\title{
Bacteremia Due to a Multidrug Resistant Staphylococcus aureus: A Case Report of Unsuccessful Treatment
}

\author{
Aliehsan Heidari ${ }^{1}$, Enayatollah Kalantar ${ }^{2}$, Fatima Rahimi ${ }^{3}$, Yahya Yaghoobi ${ }^{4}$, Sara Ghozati ${ }^{3,}{ }^{,}$, \\ Mohammad Hossein Dehghan ${ }^{3}$ and Parviz Fallah ${ }^{3}$ \\ ${ }^{1}$ Department of Parasitology, School of Medicine, Alborz University of Medical Sciences, Karaj, Iran \\ ${ }^{2}$ Department of Microbiology, School of Medicine, Alborz University of Medical Sciences, Karaj, Iran \\ ${ }^{3}$ Rajaei Hospital, Alborz University of Medical Sciences, Karaj, Iran \\ ${ }^{4}$ Department of Infectious Diseases, Rajaei Hospital, Alborz University of Medical Sciences, Karaj, Iran \\ "Corresponding author: Rajaei Hospital, Alborz University of Medical Sciences, Karaj, Iran. Tel: +98-9123686318, Email: saraghozati@yahoo.com
}

Received 2018 March 17; Revised 2018 July 27; Accepted 2018 August 28.

\begin{abstract}
Vancomycin-resistant Staphylococcus aureus has emerged as a multidrug-resistant pathogen responsible for mortality, particularly among ICU patients. This study reports a case of bacteremia associated with mortality in ICU ward in a major referral hospital in Karaj. Although antibiotic treatment was started, the patient died. This report describes the clinical course of the illness. To the best of our knowledge, no case of multidrug-resistant Staphylococcus aureus has been reported previously in Karaj.
\end{abstract}

Keywords: Bacteremia, Vancomycin-Resistant, Staphylococcus aureus, Unsuccessful Treatment

\section{Introduction}

Bacteremia is one of the most invasive diseases and responsible for severe infections and has a high impact on public health problems all over the world. Various bacteria, particularly Gram-positive bacteria, are often responsible for such severe and difficult to treat infections, which are characterized by high mortality rates in hospital settings $(1,2)$. Therefore, bacteremia due to this kind of bacteria combined with resistances to treatment options has become more common worldwide and has been listed as a particular risk to human health by World Health Organization, the European Center for Disease Control, and the US Center for Disease Control (3-5).

The emergence of antimicrobial-resistant organisms, including vancomycin-resistant Staphylococcus aureus, is a challenge to clinicians due to the lack of effective antimicrobial agents available against them and associated infection control implications.

We describe the characteristics of a patient with Staphylococcus aureus bacteremia in an intensive care unit in a teaching hospital of a developing country, a case of bacteremia caused by a high-level vancomycin-resistant.

\section{Case Presentation}

A forty-seven-year-old female patient had diabetes, blood pressure, hyperlipidemia, Adrenalectomy because of Pheochromocytoma, which was under chemotherapy, referred to the emergency department with abdominal pain and her blood sugar was 370 . Primary vital symptoms of the patient at the time of arrival were as follows: BP: 100/66, HR: 60, RR: 20, and T:37. Then after taking the first steps, she received albumin and blood glucose control, the patients feel better and after 48 hours, she was discharged.

Twenty-five days after she was discharged, again, she was admitted to the emergency ward with shortness of breath, high blood sugar, dyspnea and abdominal pain, weakness and fever, edema, and decreased consciousness.

Early signs and symptoms of the patient were BP: 149/88, HR: 123, RR: 28, and T:39. She was diagnosed with sepsis and referred to the infectious ward and the treatment with imipenem and ciprofloxetin was initiated.

Blood culture was taken before the application of antibiotics and anesthesia and infectious consultation for the patient. Treatment and care processes were done to reduce patient fever, alertness, control blood glucose, reduce the patient's edema, and control electrolytes as per physician suggestions. The patient was discharged after two days of hospitalization and treatment and had a good clinical outcome with a resolution of all symptoms.

Unfortunately, after a week, the patient again referred to the emergency department for the third time with reduced alertness, hand and legs generalized edema, shortness of breath, dyspnea and high fever. The signs and symp- 
toms of the patient are as follows: BS: 296, BP: 142/94, HR: 13, RR: 28 and T: 39 . She was diagnosed with sepsis again and the treatment started with Meropenem. However, after an hour, she showed respiratory depression and shortness of breath, the recovery code was implemented for the patient and transferred to ICU. After the consultation, cardiography eco, brain scan, Doppler vascular ultrasound and lung scan all were done which were normal. However, blood culture was positive for Staphylococcus aureus. The isolate was tested for its antimicrobial susceptibility pattern using CLSI guideline (6). The isolate was resistant to co-trimoxazole, vancomycin, ampicillin, ciprofloxacin, ceftizoxim, erythromycin, gentamicin, amikacin, and ceftazidime. She ultimately passed away on day three.

\section{Discussion}

Many scientists believe vancomycin-resistant Staphylococcus aureus is an important nosocomial pathogen and in patients with bacteremia, has very bad outcomes and increases mortality and length of hospital stay $(7,8)$. Therefore, preventing nosocomial multidrug bacterial infections should be a priority in health care.

Many factors like age, admission to an intensive care unit, mechanical ventilation, trauma, and prolonged hospitalization are the important risk factors for many hospital-acquired bacterial infections. One important aspect of our patients was that Staphylococcus aureus caused bacteremia and this isolate was highly resistant to various antibiotics, particularly vancomycin, emphasizing that Staphylococcus aureus infections appear to be very difficult to eradicate and can be taken into account as a source of severe and life-threatening illness. Therefore, health care providers need to be aware of the clinical significance of this recognized pathogen. Hence, alternative therapeutic options are urgently needed to treat these kinds of patients.

\section{Acknowledgments}

We would like to thank medical record staff of Rajaie Hospital for their collaboration. The authors received no funding.

\section{Footnotes}

Conflict of Interests: The authors declare that there is no conflict of interest regarding the publication of this paper. Funding/Support: There is no funding for this case report. Patient Consent: Informed consent was obtained from the patient's parent for the publication of this case report.

\section{References}

1. Doernberg SB, Lodise TP, Thaden JT, Munita JM, Cosgrove SE, Arias CA, et al. Gram-positive bacterial infections: Research priorities, accomplishments, and future directions of the antibacterial resistance leadership group. Clin Infect Dis. 2017;64(suppl_1):S24-9. doi: 10.1093/cid/ciw828. [PubMed: 28350900]. [PubMed Central: PMC5850444].

2. Menichetti F. Current and emerging serious Gram-positive infections. Clin Microbiol Infect. 2005;11 Suppl 3:22-8. doi: 10.1111/j.14690691.2005.01138.x. [PubMed: 15811021].

3. World Health Organization. Antimicrobial resistance: Global report on surveillance. World Health Organization; 2014.

4. European Centre for Disease Prevention and Control. Proposals for draft EU guidelines on the prudent use of antimicrobials in human medicine. 2016.

5. Centers for Disease Control and Prevention. Antibiotic resistance threats in the United States. CDCP; 2013.114 p.

6. Clinical and Laboratory Standards Institute. Performance standards for antimicrobial susceptibility testing; twenty-fifth informational supplement," CLSI Document M100-S25. Wayne, Pa, USA: CLSI; 2015.

7. Bassetti M, Peghin M, Trecarichi EM, Carnelutti A, Righi E, Del Giacomo $P$, et al. Characteristics of Staphylococcus aureus bacteraemia and predictors of early and late mortality. PLoS One. 2017;12(2). e0170236. doi: 10.1371/journal.pone.0170236. [PubMed: 28152067]. [PubMed Central: PMC5289427].

8. Soriano A, Martinez JA, Mensa J, Marco F, Almela M, MorenoMartinez A, et al. Pathogenic significance of methicillin resistance for patients with Staphylococcus aureus bacteremia. Clin Infect Dis. 2000;30(2):368-73. doi: 10.1086/313650. [PubMed: 10671343]. 